\title{
Isolation and Molecular Characterization of a New CRT Binding Factor Gene from Capsella bursa-pastoris
}

\author{
Xinglong Wang ${ }^{\dagger}$ Li Liù, Sixiu Liù, Xiaoqing Sun', Zhongxiang Deng \\ Yan Pi ${ }^{\dagger}$, Xiaofen Sun ${ }^{\dagger} *$ and Kexuan Tang ${ }^{\dagger, * * *}$ \\ ${ }^{\dagger}$ State Key Laboratory of Genetic Engineering, School of Life Sciences, Fudan-SJTU-Nottingham Plant Biotechnology R\&D Center, \\ Morgan-Tan International Center for Life Sciences, Fudan University, Shanghai 200433, People's Republic of China \\ ${ }^{\ddagger}$ Plant Biotechnology Research Center, Fudan-SJTU-Nottingham Plant Biotechnology R\&D Center, \\ School of Agriculture and Biology, Shanghai Jiaotong University, Shanghai 200030, People's Republic of China
}

Received 3 January 2004, Accepted 17 February 2004

\begin{abstract}
A new CRT binding factor (CBF) gene designated $\mathrm{Cbcbf25}$ was cloned from Capsella bursa-pastoris, a wild grass, by the rapid amplification of cDNA ends (RACE). The fulllength cDNA of $C b c b f 25$ was 898 bp with a 669 bp open reading frame (ORF) encoding a putative DRE/CRT (LTRE)-binding protein of 223 amino acids. The predicted CbCBF25 protein contained a potential nuclear localization signal (NLS) in its N-terminal region followed by an AP2 DNA-binding motif and a possible acidic activation domain in the $\mathrm{C}$-terminal region. Bioinformatic analysis revealed that $C b c b f 25$ has a high level of similarity with other CBF genes like $c b f 1, c b f 2$, and $c b f 3$ from Arabidopsis thaliana, and Bncbf5, Bncbf7, Bncbf16, and Bncbf17 from Brassica napus. A cold acclimation assay showed that Cbcbf 25 was expressed immediately after cold triggering, but this expression was transient, suggesting that it concerns cold acclimation. Our study implies that $\boldsymbol{C b c b f 2 5}$ is an analogue of other CBF genes and may participate in cold-response, by for example, controlling the expression of cold-regulated genes or increasing the freezing tolerance of plants.
\end{abstract}

Keywords: AP2 domain, Capsella bursa-pastoris, Cbcbf25, CBF, RACE

Database Accession No.: AY491498

\footnotetext{
*To whom correspondence should be addressed.

Tel: 86-21-65642772; Fax: +86-21-65643552

E-mail: kxtang1@yahoo.com or kxtang1@sohu.com or xfsun1@sohu.com
}

\section{Introduction}

Cold adversely affects the growth and productivity of plants (Thomashow, 1999), and many plants have the ability to respond and adapt to low temperatures. Previous studies have shown that cold can signal trigger a cascade of transcriptional regulation in plants, and that cold-regulated gene expression is a critical factor of chill tolerance (Gong et al., 2002; Hsieh et al., 2002) and in the cold acclimation of plants (Knight et al., 1999; Thomashow, 1999; Tahtiharju and Palva, 2001). These cold-responsive genes encode a diverse array of proteins, such as, enzymes involved in respiration and in the metabolism of carbohydrates, lipids phenylpropanoids and antioxidants, molecular chaperones, antifreezing proteins, and others, which are presumed to impart tolerance to dehydration caused by freezing (Mohapatra et al., 1989; Guy, 1990; Thomashow, 1999).

The CBF (CRT binding factor) cold-responsive pathway is an integral component of the cold acclimation response (Shinozaki and Yamaguchi-Shinozaki, 1996; Thomashow, 1999, 2001; Fowler and Thomashow, 2002). The Arabidopsis CBF family (cbf1, cbf2, and cbf3) (Gilmour et al., 1992; Stockinger et al., 1997; Liu et al., 1998; Medina et al., 1999), the winter Brassica napus CBF family (Bncbf5, Bncbf7, Bncbf16, and Bncbf17) (Gao et al., 2002), and several other CBF genes from Lycopersicon esculentum, Oryza sativa and barley (Jagglo-Ottosen et al., 2001; Choi et al., 2002) have been identified and isolated, and CBF proteins have been found to share some common characteristic motifs such as NLSs (nuclear localization signals), an AP2 DNA-binding domain, and acidic C-terminal fragments. The CBF proteins were found to bind the CRT (C-repeat) elements (cis-regulator elements in the promoters of cold-responsive genes such as Cor78 and Cor15a), and to activate the downstream genes (Thomashow, 1999). Further study revealed that the CRT 
element regulates gene expression in an ABA-independent pathway (Yamaguchi-Shinozaki and Shinozaki, 1994). The over expression of cbfl in Arabidopsis was found to increase the freezing tolerance of transformed plants in the absence of a prior cold treatment (Jagglo-Ottosen et al., 1998; Medina et al., 1999). In fact, all earlier studies have shown that CBF plays a positive role in the cold acclimation process, by controlling cold-responsive gene expression and by increasing freezing tolerance.

Capsella bursa-pastoris is a wild grass, which belongs to the Cruciferae like Arabidopsis and B. napus. It is considered to be a potential vegetable crop and can grow and set seeds normally at low temperatures, suggesting that it possesses a cold-acclimation pathway. Thus, we believed that $C$. bursapastoris was likely to contain CBF-like genes, and if so, the cloning of these genes could be useful for the study of the mechanism of cold tolerance in C. bursa-pastoris. To date no study has reported on the cloning of CBF genes from $C$. bursa-pastoris. In this paper, we describe the cloning and molecular characterization of a new CBF gene, named Cbcbf25, from C. bursa-pastoris by using RACE technology. Bioinformatic analysis and a cold acclimation assay revealed that the $C$. bursa-pastoris $\mathrm{CBF}$ gene (Cbcbf25) strongly resembles $\mathrm{CBF}$ genes from other species, and that this is relevant to cold acclimation.

\section{Materials and Methods}

Materials Young leaves of C. bursa-pastoris, collected from the Fudan University campus, Shanghai, China, were used for RNA isolation. Molecular cloning of Cbcbf 25 from C. bursa-pastoris was carried out using a RACE PCR Kit (GIBCO BRL, Gaithersburg, USA) and a SMART ${ }^{\mathrm{TM}}$ RACE cDNA Amplification Kit (Clontech, Palo Alto, USA). Primers used in the study, shown in Table 1, were synthesized by the Shanghai Sangon Biotechnological Company, and DNA sequencing was performed by the Shanghai Genetech Biotechnological Company. The pGEM T-easy vector was purchased from Promega (Madison, USA). All other chemicals used were of analytical grade.

\section{Methods}

Isolation of total RNA Approximately $1.0 \mathrm{~g}$ of freshly harvested young leaves of $C$. bursa-pastoris were allowed to stand at $4^{\circ} \mathrm{C}$ for $12 \mathrm{~h}$ and then homogenized in liquid nitrogen. Total RNA was extracted using TRIZOL reagent according to the manufacturer's

Table 1. Primers used for the cloning of Cbcbf 25

\begin{tabular}{ll}
\hline \multicolumn{1}{c}{ Primers } & \multicolumn{1}{c}{ Primer sequence $\left(5^{\prime} \rightarrow 3^{\prime}\right)$} \\
\hline CBF\# 5'GSP1 & 5'-AGTATTCCATTGTACGGACGGAGG-3' \\
CBF\# 5'GSP2 & 5'-ATCGCTCTGTTCCGGCGTCAC-3' \\
3'jcCBF\#GSP1 & 5'-AGATGGCTGCTCGTGCTCACGAC-3' \\
3'jcCBF\#GSP2 & 5'-ACGACTACGGCTGCGACTACGAC3' \\
FCBCBF & 5'-ACGCGGGAACCTTATCCAAACAG-3' \\
RCBCBF & 5'-TTTACATTCGTTTCTCACACTAGCCAC-3' \\
\hline
\end{tabular}

instructions (GIBCO BRL, Gaithersburg, USA).

5' RACE of $\boldsymbol{C b} \boldsymbol{c} \boldsymbol{b} \boldsymbol{2 5}$ According to the protocol of the SMART ${ }^{\mathrm{TM}}$ RACE cDNA Amplification kit (Clontech), about $100 \mathrm{ng}$ of total RNA was reverse transcribed with primer 5'-CDS primer coupled with (dC) tailing and SMART II A oligo, which annealed to the tail of the RNA and served as an extended template for PowerScript RT. The reverse primer used for the cloning of a partial coding sequence of $C b c b f 25$ was designed and synthesized according to the conservative regions of the CBF gene sequences of Arabidopsis and $B$. napus in GenBank. First round PCR was performed with CBF5'\#GSP1 as the reverse primer and Universal Primer A Mix (UPM) as the forward primer. PCR was carried out in a total volume of $50 \mu \mathrm{l}$ containing $2.5 \mu \mathrm{l}$ cDNA, 10 pmol primer CBF5'\#GSP1, $5 \mu$ l UPM, $41.5 \mu$ l Master Mix (34.5 $\mu$ l PCR-Grade Water, $5 \mu \mathrm{l} 10 \times$ Advantage 2 PCR buffer, $10 \mathrm{mmol}$ dNTP Mix, $1 \mu \mathrm{l} 50 \times$ Advantage 2 Polymerase Mix) under the following condition: the template was firstly denatured at $94^{\circ} \mathrm{C}$ for $3 \mathrm{~min}$ and then subjected to 35 cycles of amplification $\left(94^{\circ} \mathrm{C}\right.$ for $1 \mathrm{~min}, 59^{\circ} \mathrm{C}$ for $1 \mathrm{~min}, 72^{\circ} \mathrm{C}$ for $1 \mathrm{~min}$ ) followed by $10 \mathrm{~min}$ at $72^{\circ} \mathrm{C}$. Subsequently, nested PCR was performed under normal PCR reaction conditions using CBF5'\#GSP2 as reverse primer and the Nested Universal Primer A (NUP, 5'-AAGCAGTGGTATCAACG CAGAGT-3') as forward primer. The nested-PCR product (CBCBF25a, about $550 \mathrm{bp}$ ) was purified and cloned into pGEM-T vector (Promega), and sequenced. A Blast Search revealed that the 5'RACE product CBCBF25a had high similarity with CBF genes from Arabidopsis and B. napus. Subsequently, the 3' RACE primer 3'jcCBF\#GSP1 and the nested PCR primer 3'jcCBF\#GSP2 were designed and synthesized according to the alignment results of CBCBF25a to clone the 3' end of Cbcbf 25.

3' RACE of $\boldsymbol{C b} \boldsymbol{c b f} \mathbf{2 5}$ According to the protocol of the 3' RACE System for Rapid the Amplification of cDNA ends (GIBCO BRL), an aliquot of isolated $100 \mathrm{ng}$ RNA was reverse transcribed using the cDNA synthesis primer, AP (5'-GGCCACGCGTCGACTAGTAC $(\mathrm{T})_{17}-3$ '), provided with the kit (GIBCO BRL). The gene-specific primer 3 'jcCBF\#GSP1 was used as the forward primer and Abridged Universal Amplification Primer (AUAP, 5'-GGCCACGC GTCGACTAGTAC-3') was used as the reverse primer to amplify the $3^{\prime}$ end cDNA. PCR was performed in a total volume of $50 \mu \mathrm{l}$ containing $2 \mu \mathrm{l}$ cDNA, $10 \mathrm{pmol}$ of each of the primers 3'jcCBF\#GSP1 and AUAP, 10 mmol dNTPs, $1 \times$ cDNA reaction buffer and $8 \mathrm{U}$ Taq polymerase using 35 amplification cycles $\left(94^{\circ} \mathrm{C}\right.$ for $1 \mathrm{~min}, 60^{\circ} \mathrm{C}$ for $1 \mathrm{~min}$, and $72^{\circ} \mathrm{C}$ for $1 \mathrm{~min}$ ). Nested PCR was carried out in a total volume of $50 \mu \mathrm{l}$ containing $1 \mu \mathrm{l}$ of the product of the first round $3^{\prime}$ RACE-PCR using the same PCR conditions. The nested-PCR product (CBCBF25b, about $400 \mathrm{bp}$ ) was purified, cloned into pGEM-T vector (Promega), and sequenced.

Generation of the full-length cDNA of $\boldsymbol{C b c b f 2 5}$ By comparing and aligning the sequences of the 5'RACE product CBCBF25a and the 3' RACE product CBCBF25b, the full-length cDNA sequence of $C b c b f 25$ was deduced and subsequently amplified using the primer pair, FCBCBF and RCBCBF, using a One Step PCR Kit (Takara, Shiga, Japan). RT-PCR was carried out in a total volume of $50 \mu \mathrm{l}$ containing $100 \mathrm{ng}$ RNA, $10 \mathrm{pmol}$ of each of FCBCBF and RCBCBF, 10 mmol dNTPs, $1 \times$ One Step RNA PCR buffer, 5 U 
RNase Inhibitor, 10U AMV Reverse Transcriptase XL and $10 \mathrm{U}$ AMV-Optimized Taq under the following conditions: $50^{\circ} \mathrm{C}$ for 30 min and $94^{\circ} \mathrm{C}$ for 2 min followed by 30 cycles of amplification $\left(94^{\circ} \mathrm{C}\right.$ for $30 \mathrm{sec}, 60^{\circ} \mathrm{C}$ for $30 \mathrm{sec}$ and $72^{\circ} \mathrm{C}$ for $\left.5 \mathrm{~min}\right)$. The RT-PCR product (CBCBF25c, about $850 \mathrm{bp}$ ) was purified and cloned into pGEM-T vector and then sequenced.

Sequence analysis The full-length $\mathrm{C} b c b f 25$ and associated molecular information were analyzed using Vector NTI Suite 6.0, BioEdit, and Clustal W.

Secondary and three-dimensional structure analyses The secondary structure of CbCBF25 was analyzed by SOMPA (http:// npsa-pbil.ibcp.fr/cgi-bin/npsaautomat.pl?page $=$ npsasopma.html).

Three-dimensional structure analysis and molecular modeling were performed using TOPITS (http://www.embl-heidelberg.de/ predictprotein/predictprotein.html) (data submitted to database SWISS-MODEL, SDSC1, Loopp, Superfamily, DAS, JPRED, PHD, PROF, PSIpred, PSSP, SAM-T99 and SSpro separately), Frsvr (http://www.mbi.ucla.edu/people/frsvr.html) and 123D+ (http://123d.ncifcrf.gov/).

Cold acclimation assay To determine whether the cloned Cbcbf 25 was cold-responsive, total RNA was extracted from young leaves of $C$. bursa-pastoris plants subjected to various treatments, and then subjected to semi-quantitative RT-PCR for Cbcbf 25 expression. These treatments included first growing the plant at $28^{\circ} \mathrm{C}$ for $4 \mathrm{~d}$, subsequently treating at $4^{\circ} \mathrm{C}$ for $0.5,1,1.5,2$ or $4 \mathrm{~h}$, and then at $28^{\circ} \mathrm{C}$ for $2 \mathrm{~d}$ or $4 \mathrm{~d}$, respectively. RT-PCRs using a One Step PCR Kit (Takara, Japan) were performed at $50^{\circ} \mathrm{C}$ for $30 \mathrm{~min}$ followed by 30 cycles of amplification $\left(94^{\circ} \mathrm{C}\right.$ for $1 \mathrm{~min}, 62^{\circ} \mathrm{C}$ for $1 \mathrm{~min}$ and $72^{\circ} \mathrm{C}$ for $1 \mathrm{~min}$ ), using gene-specific primers FCBCBF and RCBCBF. Two primers were also used to amplify ubiquitin gene (AY189972) from $1 \mathrm{nt}$ to $250 \mathrm{nt}$ as a positive control for semiquantitative RT-PCR.

\section{Results and Discussion}

Isolation and characterization of the full-length cDNA of Cbcbf25 Using the RT-PCR method and primers mentioned above, the full-length cDNA of Cbcbf25 (Genbank accession No. AY491498) was obtained; this was subsequently confirmed by sequencing. The full-length cDNA was $898 \mathrm{bp}$ long; a size similar to CBF genes from A. thaliana (Cbfl, $C b f 2, C b f 3)$ and B. napus (Bncbf5, Bncbf7, Bncbfl6 and Bncbf17), and contained a $669 \mathrm{bp}$ open reading frame encoding a putative DRE/CRT (LTRE)-binding protein of 223 amino acids with a calculated isoelectric point (pI) of 4.84 and a calculated molecular weight of ca. $25 \mathrm{kDa}$ (Fig. 1).

Transmembrane helix prediction (TMHMMv2.0, http:// www.cbs.dtu.dk/services/TMHMM-2.0/) showed that there were no transmembrane helices in the deduced $\mathrm{CbCBF} 25$

\begin{tabular}{|c|c|c|c|c|c|c|c|c|c|c|}
\hline 1 & & $\triangle C G C G G$ & GACCT & TATCCA & $\triangle A C A G A$ & $G C A G A C$ & TGAGTT & AGTCCA & A AGTCC & $\sin 0$ \\
\hline 1 & $M$ & $s \quad s$ & $F$ & $S \quad A$ & $F \quad S$ & E $M$ & $F \quad G$ & $s \quad 0$ & $Y \quad E$ & $s$ \\
\hline 61 & TGAAC & ГCATCA & TCATTC & TCTGCT & TTCTCT & GASATG & TTTGGC & TCCGAT & TACGAG & 1010 \\
\hline & $\mathrm{G} \quad \mathrm{s}$ & $\mathrm{s} \quad \mathrm{G}$ & $G \quad G$ & $\square \quad \gamma$ & C $\quad P$ & $\begin{array}{ll}T & \mathrm{~L}\end{array}$ & A $\quad T$ & $s \quad c$ & P $\quad K$ & K \\
\hline 21 & ICT & TCAGGC & GGCGGA & GATTAT & TGTCCT & ACGCTT & $\mathrm{GCCACG}$ & AGCTGT & CCCAAG & AADCC \\
\hline 41 & G & $\mathrm{R}$ & $\mathrm{K}$ & $\mathrm{R}$ & $\mathrm{R}$ & $\mathrm{H}$ & $Y$ & $\mathrm{R}$ & $\mathrm{R}$ & $Q$ \\
\hline 81 & SCGGGT & CGGAAG & ASGTTT & CGCGAG & $\triangle \mathrm{CTCGT}$ & $\mathrm{CACCCG}$ & ATTTAC & AGAGGA & GTTCGT & CAAAGC \\
\hline 61 & $s$ & G K & $w \quad v$ & $E$ & L $\quad R$ & $\mathrm{P}$ & N $\quad K$ & $\mathrm{~K}$ & R $\quad$ I & W L \\
\hline & $\mathrm{ACTC}$ À & GGTAMG & TGGGTC & TCTGAG & CTAMGA & $\mathrm{GAGCCA}$ & AMCAAA & $\triangle A A B C T$ & $\grave{A} G G A T T$ & TGGCT \\
\hline 81 & $\mathrm{~T}$ & $Q$ & $\mathrm{~A}$ & E $M$ & A $\mathrm{A}$ & $\mathrm{R}$ & H $\quad \mathrm{D}$ & $v$ & A & $\mathrm{T}$ \\
\hline 01 & $\mathrm{GCaCT}$ & TTCCA & $\triangle \mathrm{ACGCC}$ & GAGATG & GCTGCT & CGTGCT & $C A C G A C$ & GTCGCC & GCCATT & $\mathrm{ACCCT}$ \\
\hline 101 & $\mathbf{G}$ & R & C & L N & $F$ & $\mathrm{D}$ & A & $\mathrm{R}$ & R I & $\mathrm{P} \quad \mathrm{E}$ \\
\hline 61 & $\mathrm{GGC}$ & AGGTCA & GCCTGT & CTCAAT & TTCGCC & GACTCG & GCTTGG & CGGCTA & AGGATC & CCGGA \\
\hline 121 & $T$ & $c$ & $K$ & $\begin{array}{ll}1 & Q \\
\end{array}$ & K & A & $E$ & $\mathrm{~A}$ & $\mathrm{~A}$ & $Q$ \\
\hline & $\mathrm{CA}$ & TGC & $\mathrm{AAC}$ & $\mathrm{ATC}$ & $\mathrm{ASG}$ & ;CT & GAS & GCCTTG & GCTTTT & $\mathrm{CAs}$ \\
\hline & $M$ & G & $\mathrm{T}$ & $\mathrm{T}$ & $A$ & $\mathrm{~T}$ & $\mathrm{D}$ & $\mathrm{H}$ & $\mathrm{D}$ & $\mathrm{K}$ \\
\hline 81 & & GGT & $\triangle C G$ & $A C G$ & GCG & $\mathrm{ACG}$ & $\mathrm{ACT}$ & CATGGC & CTTGAC & $A B G$ \\
\hline 161 & $T$ & $M$ & $E$ & $T$ & $T$ & $Q$ & $\mathrm{D}$ & $S \quad L$ & $\begin{array}{ll}Y & 1 \\
\end{array}$ & $\mathrm{D}$ \\
\hline 41 & $\mathrm{ACG}$ & ATGGTG & GAAGCT & $\mathrm{ACTC}$ & $\mathrm{ACG}$ & GAA & AGC & TCGTTA & TATATA & $\mathrm{GAC}$ \\
\hline & $\mathrm{A}$ & $\mathrm{M} \quad \mathrm{F}$ & G $M$ & $\mathrm{P}$ & $\mathrm{F}$ & $\mathrm{D}$ & $M$ & E $\quad G$ & $\mathrm{M} \quad \mathrm{L}$ & $\mathrm{L}$ \\
\hline & $\mathrm{CA}$ & ATGTTC & GGGATG & $\mathrm{CCG}$ & TTTTTG & GATAGT & $\mathrm{ATG}$ & GAMGGG & ATGCTT & TTGCC \\
\hline & $\mathrm{P}$ & $s \quad v$ & Q W & $N$ & $\mathrm{~N} \quad \mathrm{Y}$ & D $\quad \mathrm{V}$ & $\mathrm{D}$ & $\mathrm{D}$ & D $\quad$ L & $s$ \\
\hline & $C G$ & TCCGTA & CAATGG & AATACT & AACTAT & GACGTC & GACGGC & GATGAT & GACTTG & TCGCT \\
\hline & $s$ & $\stackrel{\mathrm{Y}}{\longrightarrow}$ & & & & & & & & \\
\hline & TGGiGT & TATTAA & TATTTG & ATTATT & АTTATT & ATATTG & TTTTAT & TTTTTC & CATTTT & TGGTA \\
\hline 81 & TATAGT & TTTTTA & TATGTT & AGATCC & TTTTTA & TAGAAA & CGGATC & TTCTTA & ATTTTT & GTGGC \\
\hline & AGTGTG & AGASAC & GASTGT & AATGA & CTCAGT & AAAGGA & GGTTGT & CAAAA & AAAAA & AAAA \\
\hline & 19 & & & & & & & & & \\
\hline
\end{tabular}

Fig. 1. The nucleotide acid sequence of $C b c b f 25$ full-length cDNA and its deduced amino acid sequence. 
protein, which is consistent to the hydrophobicity analysis result obtained using BioEdit software. According to the result obtained from the PROSITE database (http://www.Expasy.org /prosite/) and InterProScan (http://www.ebi.ac.uk/interpro/ scan.html), CbCBF25 shows high similarity to all other CBF proteins by possessing a bipartite nuclear localization sequence (NLS) domain from $\mathrm{R}_{43}$ to $\mathrm{R}_{60}$ and an AP2 domain from $I / V_{53}$ to $S_{113}$, both of which are suspected to function as
DNA-binding domains (Ohme-Takagi and Shinshi, 1995). In order to explore the function of the AP2 domain in $\mathrm{CBF}$ proteins, we carried out further bioinformatic analysis of the AP2 domain using PROSITE, InterProScan, NCBI (http:// www.ncbi.nlm.nih.gov/blast), WU-Blast2 (http://www.dove. embl-heidelberg.de/Blast/) and FASTA (http://www2.ebi.ac.uk/ fasta3/). The results obtained showed that the AP2 domain contained some potential ATP/GTP-binding sites and two

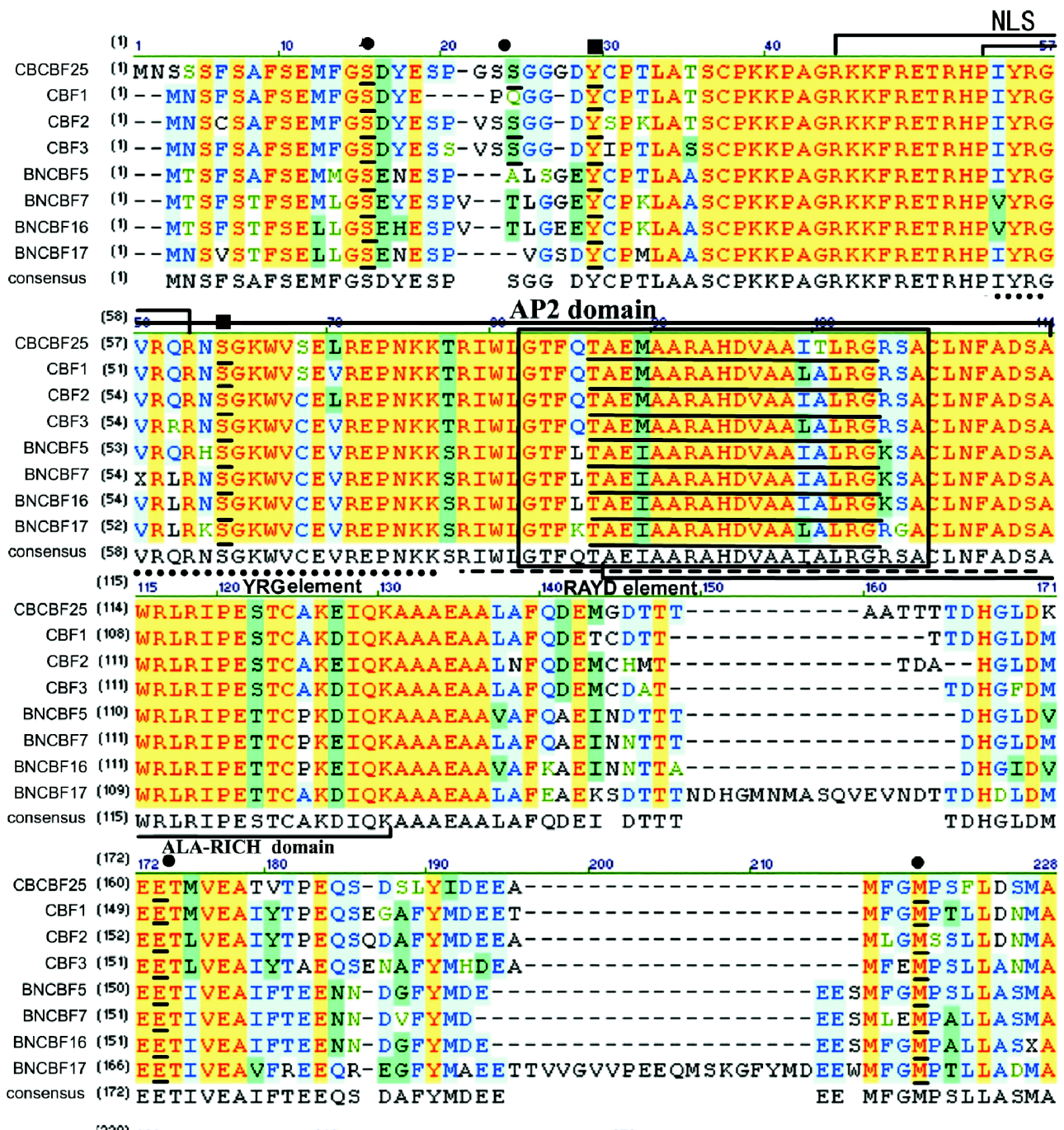

(229) 239

CBCBF25 (195) EGML L P P P SQWNTNYDVDG-D D D S SLW SY-

CBF1 (185) E GML L P P P SVOWNHNYDGEG-DGDVSLW SY-

CBF2 (188) E GML L P SP SVOWNYNF D VEG-D D D V SLW SY-

CBF3 (187) E GML L P L P SVQWNHNHE VD GD D D D V SLWSY-

BNCBF5 (186) E GML L P P P SVR F E HXYDF D G-DAXV SLW SY-

BNCBF7 (186) EGML L P P SVHF GHNYDFDG-DADV SLWSY-

BNCBF16 (187) E GML L P P S SQF GHTYDFDG-DADVSLWSY-

BNCBF17 (222) A GML L P L P SVOWGHNDDFEG-VAD INLWSY-

consensus (229) EGMLLPPP SVQWNHNYDFDG DADVSLWSY

Fig. 2. Multi-alignment of $\mathrm{CbCBF} 25$ with other known $\mathrm{CBF}$ proteins from A. thaliana (CBF1, CBF2, CBF3) and B. napus (BnCBF5, BnCBF7, BnCBF16, BnCBF17). Identical amino acid residues in the aligned sequences are indicated in yellow. Nuclear localization signals (NLS), the ALA-RICH domain, and the AP2 domain are also indicated. The conserved AP2 domain elements YRG and RAYD are marked by a dotted line and a broken line, and the amino acid residues in the RAYD conserved element predicted to form an amphipathic $\alpha$-helix and promote DNA binding are boxed. The potential ATP/GTP-binding sites are underlined by an unbroken line. Underlined amino acids indicate potential recognition sites for protein kinase $\mathrm{C}$

) and casein kinase II 
strikingly conserved elements YRG and RAYD. The YRG element, a sequence of 22 amino acids, is highly basic and contained the conserved YRG amino acid motif. It was noticeable that the RAYD element, a sequence of 36 amino acids, contained a highly conserved core region consisting of 21 amino acids, which was predicted to form an amphipathic $\alpha$-helix that might play an important role in recognizing the cis-regulator sequence and in promoting DNA binding (Okamuro et al., 1997) (Fig. 2). An ALA-Rich domain (Alanine existed comparatively mostly) from amino acid $\mathrm{A}_{86}$ to $\mathrm{K}_{129}$ was also identified (Fig. 2). According to previous studies, the ALA-Rich domain is presumed to maintain the helical structure, which is of importance in DNA binding (Bird et al., 2002). Furthermore, based on homology analysis using NCBI, WU-Blast2 and FASTA, we found that CbCBF25 contains an acidic C-terminal fragments that might act as a transcriptional activation domain and participate in protein-protein interactions, which is an essential aspect of transcriptional regulation (Hahn, 1993). Some potential recognition sites for protein kinase $\mathrm{C}$ and casein kinase II (Kennelly and Krebs, 1991) were also noticed, particularly a kinase $C$ recognition site, Ser-62, located in the AP2 DNAbinding domain (Fig. 2). Most of these sites were evolutionarily conserved among the six CBF proteins and might function in transcriptional activation via phosphorylation and dephosphorylation mechanisms (Gao et al., 2002).

Through above analyses, it can be concluded that CbCBF25 shares characteristic motifs, i.e., NLS, the AP2 domain, and acidic C-terminal fragments, with other CBF proteins; moreover, these motifs are suspected to play a critical role in DNA binding and in the activation of coldresponsive genes (Choi et al., 2002). This strongly implies that $\mathrm{CbCBF} 25$ protein may function as a trans-acting factor in cold-responsiveness in $C$. bursa-pastoris, by controlling the expressions of cold-regulated genes and by increasing the plants freezing tolerance.

Homology analysis According to the alignments of $C b f 1$, Cbf2, Cbf3, Bncbf5, Bncbf7, Bncbf16 and Bncbf17 by Clustal $\mathrm{W} / \mathrm{X}$, the regulatory sequences of $\mathrm{CBF}$ genes diverge more than the coding regions, but still retain a high level of similarity, which might explain the identical expression patterns shown by these genes (Medina et al., 1999; Gao et al., 2002). Amino acid sequence comparisons between $\mathrm{CbCBF} 25$ and other $\mathrm{CBF}$ proteins were performed using Clustal W/X and NCBI Blast. The results showed that CbCBF25 shares high homology with the CBF proteins of Arabidopsis and B. napus (Fig. 2), with identities of 83.0, 83.0, 79.6, 74.7, 73.7, 71.1, and 62.5\% to CBF1, CBF2, CBF3, BnCBF5, BnCBF7, BnCBF16, and BnCBF17, respectively (using bio-software Vector NTI suite 7.0). $\mathrm{CbCBF} 25$ was more like $\mathrm{CBF} 1$ and $\mathrm{CBF} 2$ than the other $\mathrm{CBF}$ proteins. The phylogenetic tree analysis of the six CBF proteins was consistent with the evolutionary relationship between these species (Fig. 3) (Fowler and Thomashow,
2002), suggesting that $\mathrm{CbCBF} 25$ and other $\mathrm{CBF}$ family members possess similar functions. Homologous analysis also demonstrated that the CBF gene family might have been conserved during evolution, again suggesting that they have the same functions, probably associated with survival in a harsh cold environment. The amino acid sequences of the AP2 domains of CbCBF25 showed high identity with its counterparts in other species, indicating that the AP2 domain is extremely well conserved in $\mathrm{CBF}$ proteins between different species (Fig. 2).

All our homology analyses showed that $C b c b f 25$ strikingly resembles all of the other known CBF genes in Arabidopsis and $B$. napus, and that the AP2 domain and NLS have been evolutionarily conserved, in Arabidopsis, B. napus and $C$. bursa-pastoris, which implies that they have a critical role in controlling gene expression in C. bursa-pastoris (JaggloOttosen et al., 2001).

Secondary and three-dimensional structural analyses of CbCBF25 protein Secondary structure analysis revealed that $\mathrm{CbCBF} 25$ consisted of $42 \alpha$-helices, $19 \beta$-turns jointed by 41 extended strands and 161 random coils, like other $\mathrm{CBF}$ proteins such as BnCBF5, BnCBF7, and BnCBF16 (Gao et al., 2002). Homology modeling analysis was carried out by SWISS-MODEL, SDSC1, Frsvr and 123D+ separately, while threading modeling was performed using Loopp and Superfamily. Structure analysis and molecular modeling revealed that $\mathrm{CbCBF} 25$ strongly resembles the other $\mathrm{CBF}$ proteins (Fig. 2). The presumed three-dimensional structure of CBF1, which was most similar to $\mathrm{CbCBF} 25$ in amino acid sequence, closely resembled that of $\mathrm{CbCBF} 25$. Although BnCBF17 was least similar to $\mathrm{CbCBF} 25$ in amino acid sequence and had much fewer H-bonds, helices, strands and turns, its three-dimensional structure also resembled CbCBF25 and CBF1 (Fig. 4).

All of CBFs have a common amino sequence element known as the AP2 domain, a 62-amino acid sequence encoded by the AP2 motif in the CBF genes, and which is evolutionarily conserved in plants (Stockinger et al., 1997). Molecular modeling of the AP2-domain of CbCBF25 by SWISS-MODEL showed that embedded within the AP2 domain is a large helix, four strands, and six turns, a typical feature of the AP2 domain of CBF proteins (Stockinger et al., 1997), and which might act as a DNA-binding domain (Fig. 4). The $\alpha$-helix might fit into the major groove with several of its side chains touching DNA base pairs where it recognizes and binds to the cis-regulator sequence (Ohme-Takagi and Shinshi, 1995). The structure of the protein might form a hydrophobic surface that promotes its binding with another protein of identical or similar structure to form a dimer, which would be expected to play an important role in regulating gene expression (Gong et al., 2002; Chinnusamy et al., 2003). As a result of dimerization, two recognition helices, one from each subunit of the dimer, are symmetrically extended into two adjacent major grooves of the target DNA (Mushegian and 


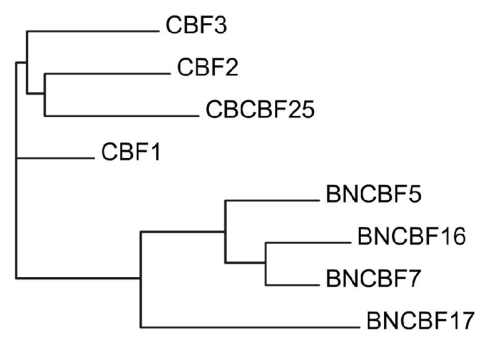

Fig. 3. Phylogenetic tree analysis of the deduced amino acid sequences of $\mathrm{CbCBF} 25$ and other $\mathrm{CBF}$ proteins from $A$. thaliana (CBF1, $\mathrm{CBF} 2, \mathrm{CBF} 3)$ and B. napus (BnCBF5, BnCBF7, BnCBF16, BnCBF17).

Koonin, 1996). Other segments in the protein, through hydrophobic interaction with the $\alpha$-helix, might create a stable structural domain that locks the $\alpha$-helix into its DNA interface. In addition, amino acid sequences outside the DNAbinding domain might affect the DNA-binding activity of AP2 domain (Mushegian and Koonin, 1996). It was also revealed that the AP2 portion of $\mathrm{CbCBF} 25$ comprises almost $30 \%$ of its protein mass. Perhaps the activation domain of AP2 domain is capable of interacting with RNA polymerase and of activating transcription through protein-protein interactions with a subunit of RNA polymerase, or interacting with other intermediary proteins (Ohme-Takagi and Shinshi, 1995).

Cold acclimation assay Semi-quantitative RT-PCR was carried out to investigate the expression of Cbcbf25 in $C$. bursa-pastoris under cold stress. The expression pattern of Cbcbf 25 was found to be similar to those of other CBFs in Arabidopsis and B. napus (Gilmour et al., 1992; Stockinger et al., 1997; Liu et al., 1998; Medina et al., 1999; Gao et al., 2002) (Fig. 5). The Cbcbf 25 transcripts increased within 30 min of exposing plants to low temperature $4^{\circ} \mathrm{C}$ and reached a maximal level after $2 \mathrm{~h}$. The low-temperature regulation of Cbcbf 25 appeared to be temporary, since the transcript levels decreased thereafter and no expression could be detected after the plant was returned to a normal temperature (28) for $4 \mathrm{~d}$. This result indicated that $C b c b f 25$ is activated by cold triggering and indeed is involved in the cold acclimate process.

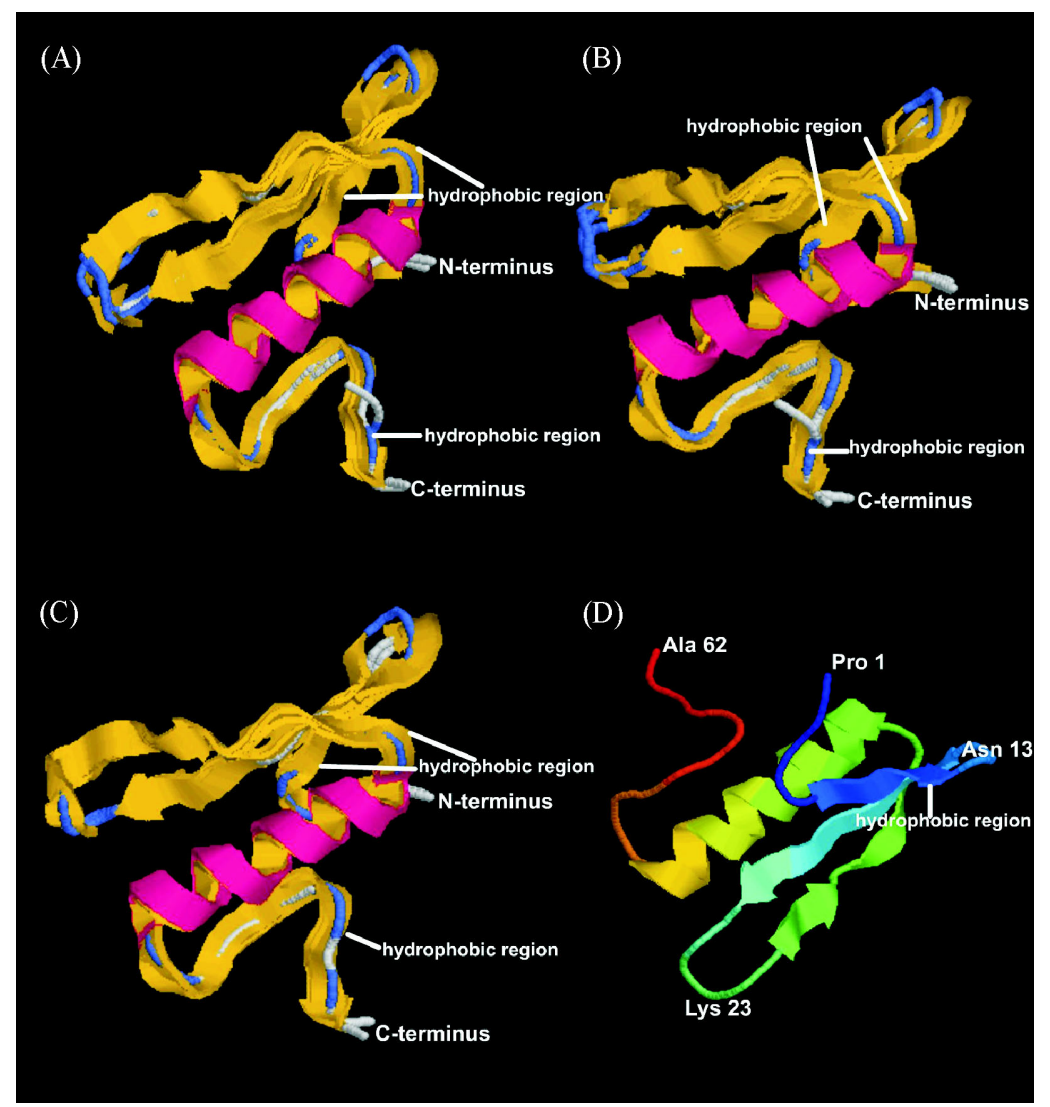

Fig. 4. Molecular modeling of $\mathrm{CBF}$ proteins using SWISS-MODEL (http://www.expasy.org/swissmod/SWISS-MODEL.html). (A) Molecular modeling of CBF1 (213 amino acids) from A. thaliana, the most similar to CbCBF25 in terms of amino acid sequence, showed that it contained 312 H-bonds, 3 helices, 16 strands and 17 turns. (B) Molecular modeling of CbCBF25 (213 amino acids) from $C$. bursa-pastoris showed that it contained 312 H-bonds, 3 helices, 15 strands and 17 turns. (C) Molecular modeling of BnCBF17 (251 amino acids) from B. napus, the least similar to CBCBF in terms of amino acid sequence, revealed that it contained $269 \mathrm{H}-\mathrm{bonds}$, 2 helices, 11 strands, and 10 turns. (D) Molecular modeling of the AP2-domain. 


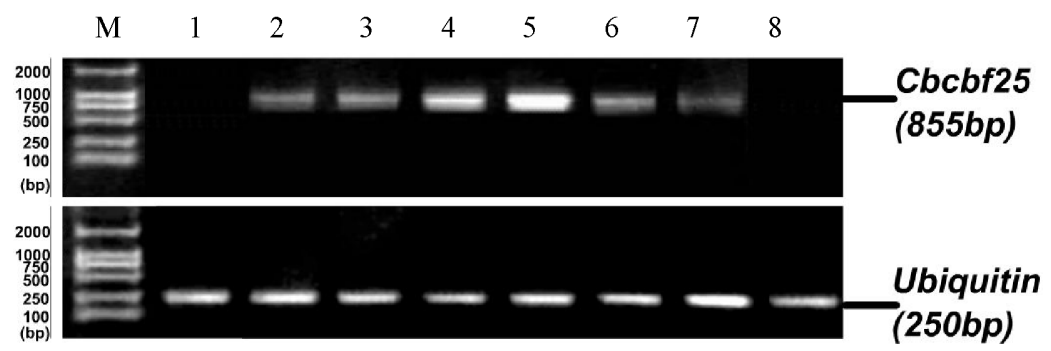

Fig. 5. Expression of $C b c b f 25$ during cold acclimation. Total RNA was extracted from young leaves of $C$. bursa-pastoris plants that had been subjected to various treatments and examined by semi-quantitative RT-PCR analysis for Cbcbf 25 expression (upper panel). The treatments include growing plant at $28^{\circ} \mathrm{C}$ for $4 \mathrm{~d}$ (Lane 1), transferring and growing the plants at $4^{\circ} \mathrm{C}$ over $0.5 \mathrm{~h}$ (Lane 2), $1 \mathrm{~h}$ (Lane 3 ), $1.5 \mathrm{~h}$ (Lane 4), $2 \mathrm{~h}$ (Lane 5), and $4 \mathrm{~h}$ (Lane 6), and then returning the plants to $28^{\circ} \mathrm{C}$ for $2 \mathrm{~d}$ (Lane 7) or $4 \mathrm{~d}$ (Lane 8). M: Molecular size marker. The ubiquitin gene (AY189972) was amplified by RT-PCR as positive control (lower panel).

Since CbCBF25 transcripts began accumulating within 30 min of exposing the plant to cold, it was supposed that a transcription factor recognizes the $C b c b f 25 \mathrm{~s}$ promoter and induces $C b c b f 25$ expression. Recently, a study in A. thaliana showed that icel (the inducer of CBF expression) acts as an upstream factor that positively regulates the expression of $c b f$ (Chinnusamy et al., 2003). It was found that Icel is expressed constitutively and that the overexpression of Icel in wild-type plants could enhance the expression of $c b f$. Another upstream gene hosl was found to encode a protein that contains a RING-finger motif, which might function in the degradation of CBF and ICE proteins (Lee et al., 2001). A study of the interaction between these genes would provide us with a better understanding of the pathway through which plants respond to cold temperature and also provide us with a useful tool for enhancing or inducing cold tolerance in plants (Thomashow, 2001).

Based on the analysis above, we suggest a potential role for CbCBF25 in the cold acclimation and freezing tolerance of $C$. bursa-pastoris. The cloning of Cbcbf 25 enables us to test whether $C b c b f 25$ can increase the freezing tolerance of transgenic plants by transferring it into non-cold-tolerant plants such as tobacco in the future.

Acknowledgments This work was funded by the Chinese National Transgenic Plant Research and Commercialization Project, the China National "863" High-Tech Program, the Chinese Ministry of Education, and the Shanghai Science and Technology Committee.

\section{References}

Bird, G. H., Lajmi, A. R. and Shin, J. A. (2002) Sequencespecific recognition of DNA by hydrophobic, Alanine-Rich mutants of the basic region/Leucine zipper motif investigated by fluorescence anisotropy. Biopolymers 65, 10-20.

Chinnusamy, V., Ohta, M., Kanrar, S., Lee, B., Hong, X., Agarwal, M. and Zhu, J. K. (2003) ICE1: a regulator of coldinduced transcriptome and freezing tolerance in Arabidopisis. Genes Dev. 17, 1043-1054.
Choi, D. W., Rodriguez, E. M. and Close, T. J. (2002) Barley CBF3 gene identification, expression pattern, and map location. Plant Physiol. 129, 1781-1787.

Fowler, S. and Thomashow, M. (2002) Arabidopsis transcriptome profiling indicates that multiple regulatory pathways are activated during cold acclimation in addition to the CBF cold response pathway. Plant Cell 14, 1675-1690.

Gao, M. J., Allard, G., Byass, L., Flanaganl, A. M. and Singh, J. (2002) Regulation and characterization of four $C B F$ transcription factors from Brassica napus. Plant Mol. Biol. 49, 459-471.

Gilmour, S. J., Artus, N. N. and Thomashow, M. F. (1992) cDNA sequence analysis and expression of two cold regulated genes of Arabidopsis thaliana. Plant Mol. Biol. 18, 13-21.

Gong, Z., Lee, H., Xiong, L., Jagendorf, A., Stevenson, B. and Zhu, J. K. (2002) RNA helicase-like protein as an early regulator of transcription factors for plant chilling and freezing tolerance. Proc. Natl. Acad. Sci. USA 99, 11507-11512.

Guy, C. L. (1990) Cold acclimation and freezing stress tolerance: Role of protein metabolism. Annu. Rev. Plant Physiol. Plant Mol. Biol. 41, 187-223.

Hahn, S. (1993) Structure and function of acidic transcription activators. Cell 72, 481-483.

Hsieh, T. H., Lee, J. T., Yang, P. T., Chiu, L. H., Charng, Y. Y., Wang, Y. C. and Chan, M. T. (2002) Heterology expression of the Arabidopsis C-repeat/dehydration response element binding factor 1 gene confers elevated tolerance to chilling and oxidative stresses in transgenic tomato. Plant Physiol. 129, 1086-1094.

Jagglo-Ottosen, K. R., Gilmour, S. J., Zarka, D. G., Schabenberger, O. and Thomashow, M. F. (1998) Arabidopsis $c b f 1$ overexpression induces $C O R$ genes and enhances freezing tolerance. Science 280, 104-106.

Jagglo-Ottosen, K. R., Kleff, S., Amundsen, K. L., Zhang, X., Haake, V., Zhang, J. Z., Deits, T. and Thomashow, M. F. (2001) Components of the Arabidopsis C-repeat/dehydrationresponsive element binding factor cold-response pathway are conserved in Brassica napus and other plant species. Plant Physiol. 127, 910-917.

Kennelly, P. J. and Krebs, E. G. (1991) Consensus sequences as substrate specificity determinants for protein kinases and protein phosphatases. J. Biol. Chem. 266, 15555-15558.

Knight, H., Veale, E. L., Warren, G. J. and Knight, M. R. (1999) The sfr6 mutation in Arabidopsis suppresses lo2-temperature 
induction of genes dependent on the CRT/DRE sequence motif. Plant Cell 11, 875-886.

Lee, H., Xiong, L., Gong, Z., Ishitani, M., Stevenson, B. and Zhu, J. K. (2001) The Arabidopsis HOS1 gene negatively regulates cold signal transduction and encodes a RING figure protein that displays cold-regulated nucleo-cytoplasmic partitioning. Genes Dev. 15, 912-924.

Liu, Q., Kasuga, M., Sakuma, Y., Abe, H., Miura, S., YamaguchiShinozaki, K. and Shinozaki, K. (1998) Two transcription factors, DREB1 and DREB2, with an EREBP/AP2 DNA binding domain separate two cellular signal transduction pathways in drought and low-temperature-responsive gene expression, respectively, in Arabidopsis. Plant Cell 10, 13911406.

Medina, J., Bargues, M., Terol, J., Perez-Alonso, M. and Salinas, J. (1999) The Arabidopsis CBF gene family is composed of three genes encoding AP2 domain-containing proteins whose expression is regulated by low temperature but not by abscisic acid or dehydration. Plant Physiol. 119, 463-470.

Mohapatra, S. S., Wolfraim, L., Poole, R. J., and Dhindsa, R. S. (1989) Molecular cloning and relationship to freezing tolerance of cold-acclimation-specific genes of alfalfa. Plant Physiol. 89, 375-380.

Mushegian, A. R. and Koonin, E. V. (1996) Sequence analysis of eukaryotic developmental proteins: ancient and novel domains. Genetics 144, 817-28.

Ohme-Takagi, M. and Shinshi, H. (1995) Ethylene-inducible DNA binding proteins that interact with an ethylene-responsive element. Plant Cell 7, 173-182.

Okamuro, J. K., Caster, B., Villarroel, R., Van Montagu, M. and Jofuku, D. K. (1997) The AP2 domain of APETALA2 defines a large new family of DNA binding proteins in Arabidopsis. Proc. Natl. Acad. Sci. USA 13, 7076-7081.

Shinozaki, K. and Yamaguchi-Shinozaki, K. (1996) Molecular responses to drought and cold stress. Curr. Opin. Biotechnol. 7, 161-167.

Stockinger, E. J., Gilmour, S. J. and Thomashow, M. F. (1997) Arabidopsis thaliana $C B F 1$ encodes an AP2 domain-containing transcriptional activator that binds to the C-repeat/DRE, a cisacting DNA regulatory element that stimulates transcription in response to low temperature and water deficit. Proc. Natl. Acad. Sci. USA 94, 1035-1040.

Tahtiharju, S. and Palva, T. (2001) Antisense inhibition of protein phosphatase 2C accelerates cold acclimation in Arabidopsis thaliana. Plant J. 26, 461-470.

Thomashow, M. (1999) Plant cold acclimation: freezing tolerance genes and regulatory mechanisms. Annu. Rev. Plant Physiol. Plant Mol. Biol. 50, 571-99.

Thomashow, M. (2001) So what's new in the field of plant cold acclimation? Lots! Plant Physiol. 125, 89-93.

Yamaguchi-Shinozaki, K. and Shinozaki, K. (1994) A novel cisacting element in an Arabidopsis gene is involved in responsiveness to drought, low-temperature, or high-salt stress. Plant Cell 6, 251-264. 